\title{
Anti-Hyperglycemic Effect of Chebulagic Acid from the Fruits of Terminalia chebula Retz
}

\author{
Yi-Na Huang ${ }^{1, \dagger}$, Dong-Dong Zhao ${ }^{2, \dagger}$, Bo Gao ${ }^{1, \dagger}$, Kai Zhong ${ }^{3, \dagger}$, Rui-Xue Zhu ${ }^{3}$, Yan Zhang ${ }^{3}$, \\ Wang-Jun Xie ${ }^{3}$, Li-Rong Jia ${ }^{3, *}$ and Hong Gao ${ }^{3, *}$
}

1 Department of Public Health, Hua Xi Medicinal Center of Sichuan University, Chengdu 610041, China; E-Mails: dir0932@ sina.com (Y.-N.H.); gaobo03@scu.edu.cn (B.G.)

2 Sichuan Academy of Medical Science \& Sichuan Provincial People's Hospital, Chengdu 610072, China; E-Mail: zhaodongd@gmail.com

3 College of Light Industry, Textile and Food Engineering, Sichuan University, Chengdu 610065, China; E-Mails: eric211@163.com (K.Z.); zhu_ruixue@126.com (R.-X.Z.); yanzhang-scu@foxmail.com (Y.Z.); gaobingchuang@ hotmail.com (W.-J.X.)

$\dagger$ These authors contributed equally to this work.

* Authors to whom correspondence should be addressed; E-Mails: jialirong @ scu.edu.cn (L.-R.J.); gao523@hotmail.com (H.G.); Tel.: +86-28-8540-5236; Fax: +86-28-8540-5137.

Received: 1 April 2012; in revised form: 14 May 2012 / Accepted: 16 May 2012 /

Published: 22 May 2012

\begin{abstract}
In the present study, we firstly compared rat intestinal $\alpha$-glucosidase inhibitory activity by different ethanol-aqueous extractions from the dried fruits of Terminalia chebula Retz. The enzymatic assay showed that the $80 \%$ ethanol extract was more potent against maltase activity than both $50 \%$ and $100 \%$ ethanol extracts. By HPLC analysis, it was determined that the $80 \%$ ethanol extract had a higher content of chebulagic acid than each of $50 \%$ or $100 \%$ ethanol extract. Next, we investigated how efficiently chebulagic acid could inhibit sugar digestion by determining the glucose level on the apical side of the Caco-2 cell monolayer. The result showed that the maltose-hydrolysis activity was down-regulated by chebulagic acid, which proved to be a reversible inhibitor of maltase in Caco-2 cells. On the other hand, chebulagic acid showed a weak inhibition of sucrose-hydrolysis activity. Meanwhile, chebulagic acid did not have an obvious influence on intestinal glucose uptake and was not effective on glucose transporters. Further animal studies revealed that the oral administration of chebulagic acid (100 mg/kg body weight) significantly reduced postprandial blood glucose levels by $11.1 \%$ in maltose-loaded Sprague-Dawley (SD) rats
\end{abstract}


compared with the control group, whereas the oral administration of chebulagic acid did not show a suppressive effect on postprandial hyperglycemia in sucrose- or glucose-loaded SD-rats. The results presented here suggest that chebulagic acid from $T$. chebula can be used to control blood glucose and manage type 2 diabetes, although clinical trials are needed.

Keywords: Terminalia chebula; chebulagic acid; $\alpha$-glucosidase inhibitor; anti-hyperglycemia

\section{Introduction}

Diabetes mellitus (DM) is a common metabolic disorder characterized by hyperglycemia, which is the main cause of complications related with micro- and macro-vascular diseases. DM is one of the three leading causes of death worldwide and constitutes a major health problem [1]. Postprandial hyperglycemia results from abnormal insulin secretion by $\beta$-cells in response to a meal, impaired hepatic glucose production, and defective glucose uptake by peripheral insulin-sensitive tissues, particularly the skeletal muscles. Therefore, control of postprandial plasma levels is critical in treatment of not only diabetic patients but also individuals with impaired glucose tolerance [2]. Mammalian intestinal $\alpha$-glucosidase (EC 3.2.1.20) is the key enzyme, which catalyzes the final step in the digestive process of carbohydrates. Hence, $\alpha$-glucosidase inhibitors can reduce postprandial blood glucose levels and absorption of starch and disaccharides [3]. Plant-derived non-nutrients were recently found to play potent roles in modulating postprandial hyperglycemia. It has been reported that food and herbs are rich sources of $\alpha$-glucosidase inhibitors [4-6].

The fruits of Terminalia chebula Retz (Combretaceae), also known as Xi-Qin-Ge in China, are a popular folk medicine for the treatment of various diseases, including digestive problems, diabetes, colic pain, chronic cough, sore throat and asthma. This plant has been studied for its biological activities, such as antioxidant [7,8], anticancer [9] and antibacterial activity [10]. Recently, T. chebula was reported to show an anti-diabetic effect [11-13]. A number of chemical compounds, including polyphenols [14], tannins [15] and triterpenoids [16], have been isolated from this plant species.

In the screening assay of $\alpha$-glucosidase inhibitory activity for medicinal plants in China, we found that the methanol extract from the fruits of $T$. chebula showed a potent maltase inhibitory activity and the active compounds, chebulanin, chebulagic acid and chebulinic acid, were identified from this plant species [17]. As a continued study, the inhibitory mode of action of chebulagic acid on the rat intestinal maltase-glucoamylase complex was determined [18]. In the present study, our objective was to evaluate the intestinal $\alpha$-glucosidase inhibitory effect by chebulagic acid from the fruits of T. chebula in vitro and in vivo. Therefore, we compared rat intestinal $\alpha$-glucosidase inhibitory activity of different ethanol-aqueous extractions and detected chebulagic acid in these extracts by HPLC analysis. Meanwhile, we determined $\alpha$-glucosidase inhibitory activity of chebulagic acid with the Caco-2 cell monolayer, together with evaluating the postprandial blood glucose lowering effect of chebulagic acid after sugar (maltose, sucrose or glucose) loading in Sprague-Dawley (SD) rats. 


\section{Results and Discussion}

\subsection{Isolation of Chebulagic Acid from T. chebula Fruits}

Extraction of phenolic compounds from $T$. chebula is generally carried out using various types of organic solvents such as $95 \%$ ethyl acetate, hot water, $70 \%$ methanol, and 95\% ethanol [19]. Ethanolic extraction of plant bioactives has displayed a higher yield compared with the aqueous extract [20]. In the present study, we compared rat intestinal $\alpha$-glucosidase inhibitory activity of different ethanol-aqueous extractions. Figure 1 shows the maltose-hydrolysis inhibitory activity of 50-100\% ethanol extracts of $T$. chebula fruits. Each of these ethanol extracts significantly inhibited the maltase activity, and the enzymatic inhibition was dose-dependent. In the same assay condition, the $\mathrm{IC}_{50}$ value of chebulagic acid was determined to be $37 \mu \mathrm{g} / \mathrm{mL}$ (data not shown). On the other hand, the $\mathrm{IC}_{50}$ values of $50 \%, 80 \%$ and $100 \%$ ethanol extracts against maltase were determined to be $173.6 \mu \mathrm{g} / \mathrm{mL}$, $51.7 \mu \mathrm{g} / \mathrm{mL}$ and $85.7 \mu \mathrm{g} / \mathrm{mL}$, respectively. Hence, this result revealed that the $80 \%$ ethanol extract was more potent regarding its effect on maltase activity than both $50 \%$ and $100 \%$ ethanol extracts.

Figure 1. Maltose-hydrolysis inhibitory activity of rat intestinal $\alpha$-glucosidase by $50-80 \%$ ethanol extracts from the fruits of Terminalia chebula Retz.

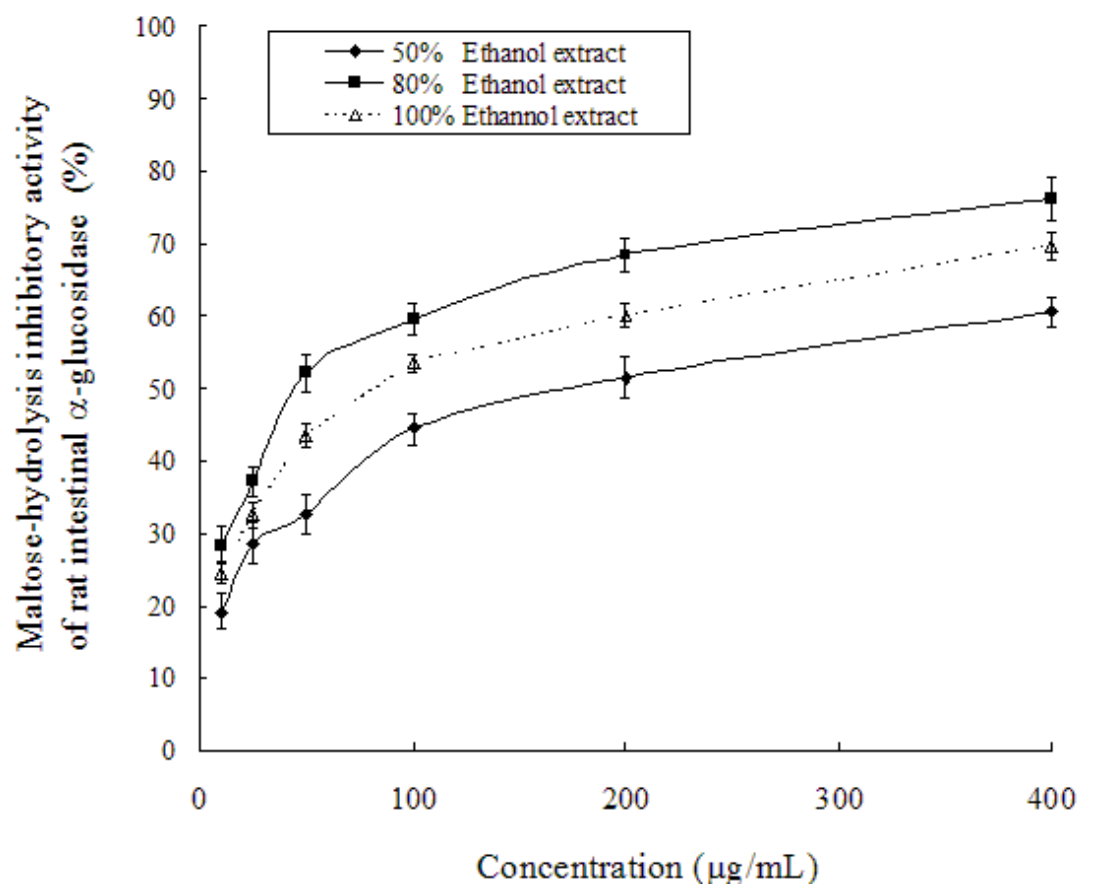

In order to ensure chebulagic acid in each ethanol extract, HPLC was used for further analysis. The compound at retention time 40.8 min was identified to be chebulagic acid, which was not found in the standard compound (Figure 2). Peak areas (mAU $\times$ Time (s)) of chebulagic acid identified in 50\%, $80 \%$ and $100 \%$ ethanol extracts were determined to be $2.55 \times 10^{4}, 6.23 \times 10^{4}$ and $4.61 \times 10^{4}$, respectively. The $80 \%$ ethanol extract contained $\sim 2.44$ - and $\sim 1.35$-times higher amounts of chebulagic acid than the $50 \%$ and $100 \%$ ethanol extracts. Therefore, chebulagic acid was prepared with our previous reported method [17] except that the dried fruit powders of $T$. chebula were extracted with $80 \%$ ethanol instead of $70 \%$ methanol, and the yield of chebulagic acid was about $7.3 \%$. The chemical 
structure of chebulagic acid was identified with ${ }^{1} \mathrm{H}-\mathrm{NMR}$ and MS and the data were compared with data in the literature [17].

Figure 2. HPLC-DAD chromatograms of 50-100\% ethanol extracts from the fruits of Terminalia chebula Retz. (A), 50\% ethanol extract; (B), 80\% ethanol extract; (C), 100\% ethanol extract.

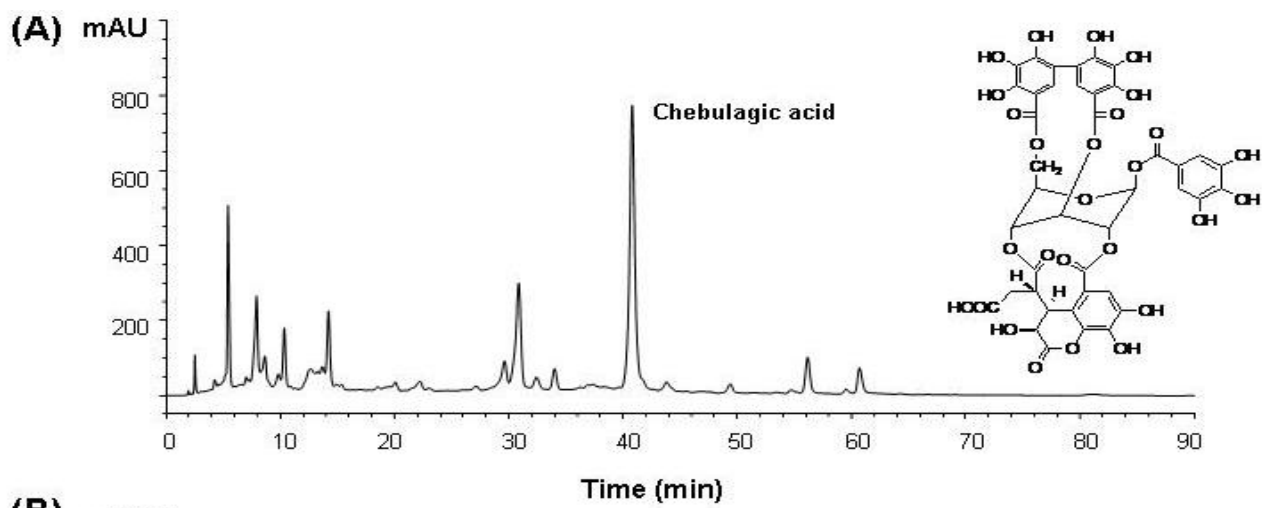

(B)

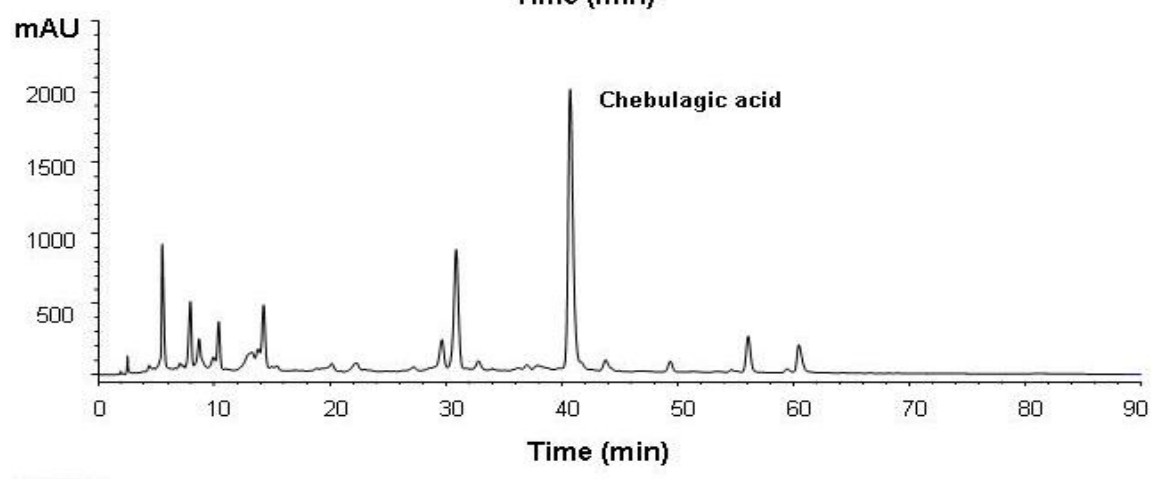

(C)

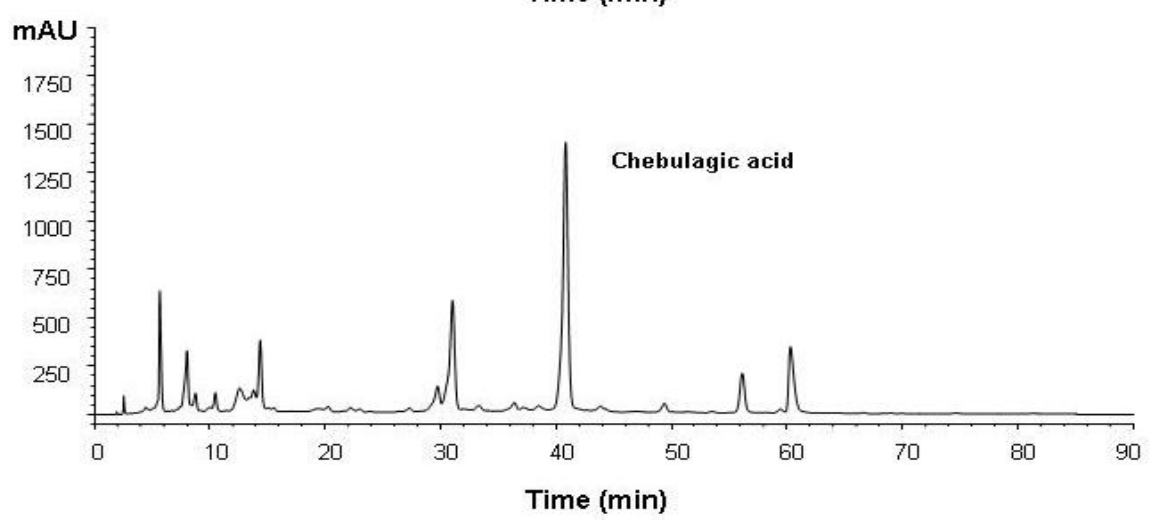

\section{2. $\alpha$-Glucosidase Inhibitory Activity in Caco-2 Cells}

At late confluency and in culture, the human colon carcinoma cell line Caco-2 expresses the same morphological characteristics and most of the functional properties of terminally differentiated small-intestinal enterocytes, including the expression of proteins involved in the terminal digestion and uptake of sugars [21]. Caco-2 cells have been widely used as a culture model of human intestinal cells in studies to determine the $\alpha$-glucosidase inhibitory effect by polyphenols [22-24]. In order to get the detailed information of chebulagic acid on mammalian intestinal $\alpha$-glucosidase inhibitory activity, we investigated how efficiently chebulagic acid could inhibit sugar digestion by determining the content of liberated glucose on the apical side of the Caco- 2 cell monolayer. 
Firstly, the effect of chebulagic acid on Caco-2 cell viability was evaluated using an MTT assay. At growth doses of $0.05-0.5 \mathrm{mM} /$ well of chebulagic acid, cell viabilities were in the ranges of 95-99\% (data not shown). This result significantly indicated a non-cytotoxic property of chebulagic acid on Caco- 2 cells. Then, Caco- 2 cells were treated with the concentrations of $0.05-0.5 \mathrm{mM} / \mathrm{well}$ levels chebulagic acid at the apical sides and the liberated glucose was measured on the apical sides. After $2 \mathrm{~h}$ of incubation, $\alpha$-glucosidse activity for the maltose hydrolysis was down-regulated, showing $73 \%$ inhibition at $0.5 \mathrm{mM} / \mathrm{well}, 62 \%$ inhibition at $0.25 \mathrm{mM} / \mathrm{well}, 51 \%$ inhibition at $0.1 \mathrm{mM} / \mathrm{well}$, and $40 \%$ inhibition at $0.05 \mathrm{mM} /$ well at the apical sides (Figure 3), respectively. PGG (1,2,3,4,6-penta- $O$ - $\beta$-D-glucose) was used as the positive control, which showed $74 \%$ inhibition of the maltose hydrolysis of $\alpha$-glucosidse activity in the Caco- 2 monolayer at the concentration of $0.5 \mathrm{mM} / \mathrm{well}$.

Figure 3. Results of the maltose hydrolysis assay with the Caco-2 monolayer in the presence of chebulagic acid (0.05-0.5 mM/well). PGG, 1,2,3,4,6-penta- $O$-galloyl- $\beta$-D-glucose $(0.5 \mathrm{mM} /$ well $)$ as a positive control.

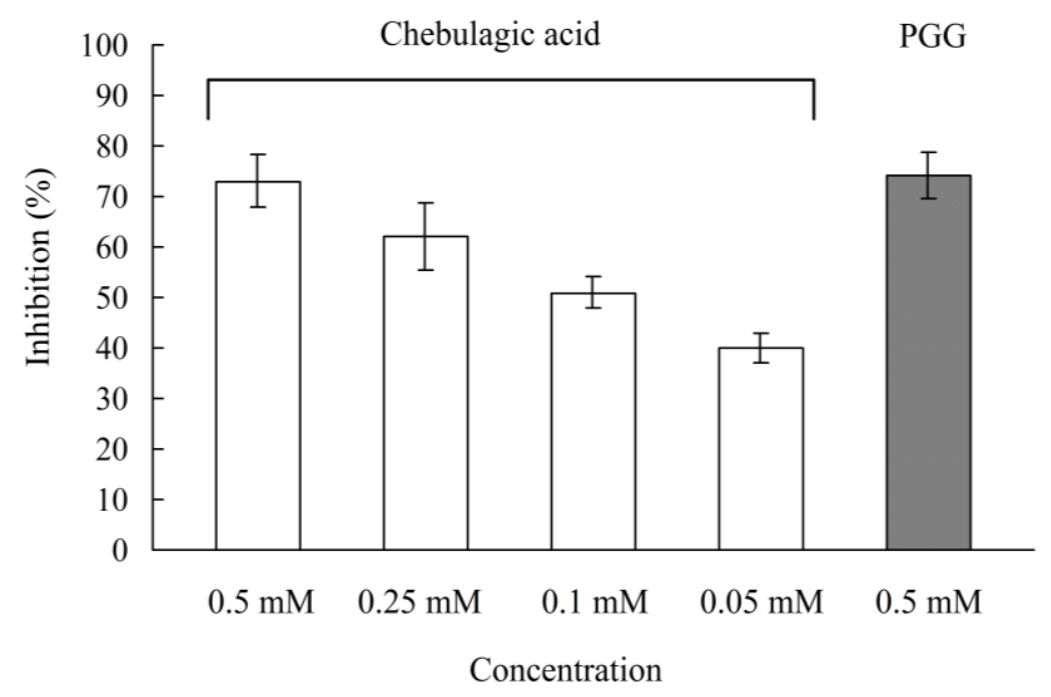

On the other hand, chebulagic acid showed a weak inhibition against sucrose-hydrolysis activity of $\alpha$-glucosidase with the Caco-2 monolayer. As shown in Figure 4, the inhibitory effect of chebulagic acid at the apical sides of the Caco-2 monolayer was determined, and the inhibitions were 20-24\% at the concentrations of $0.05-0.5 \mathrm{mM} /$ well levels. Baicalein (5,6,7-trihydroxyflavone), a potent sucrase inhibitor, showed $58 \%$ inhibition of the sucrose-hydrolysis activity of $\alpha$-glucoside in the Caco- 2 monolayer at the concentration of $0.05 \mathrm{mM} /$ well. Our previous study reported that the inhibitory influence of chebulagic acid on the maltase-glucoamylase complex was more potent than on the sucrase-isomaltase complex [18]. In agreement with the rat enzyme assay, chebulagic acid affected the Caco-2 maltase reaction more than the Caco-2 sucrase reaction. Therefore, the anti-diabetic effect of chebulagic acid might be attributed to maltose-hydrolysis inhibitory activity of intestinal $\alpha$-glucosidase, which can retard the digestion of carbohydrates resulting in mitigating postprandial hyperglycemic excursions. 
Figure 4. Results of the sucrose hydrolysis assay with the Caco-2 monolayer in the presence of chebulagic acid (0.05-0.5 mM/well). Baicalein, 5,6,7-trihydroxyflavone $(0.05 \mathrm{mM} /$ well $)$ as a positive control.

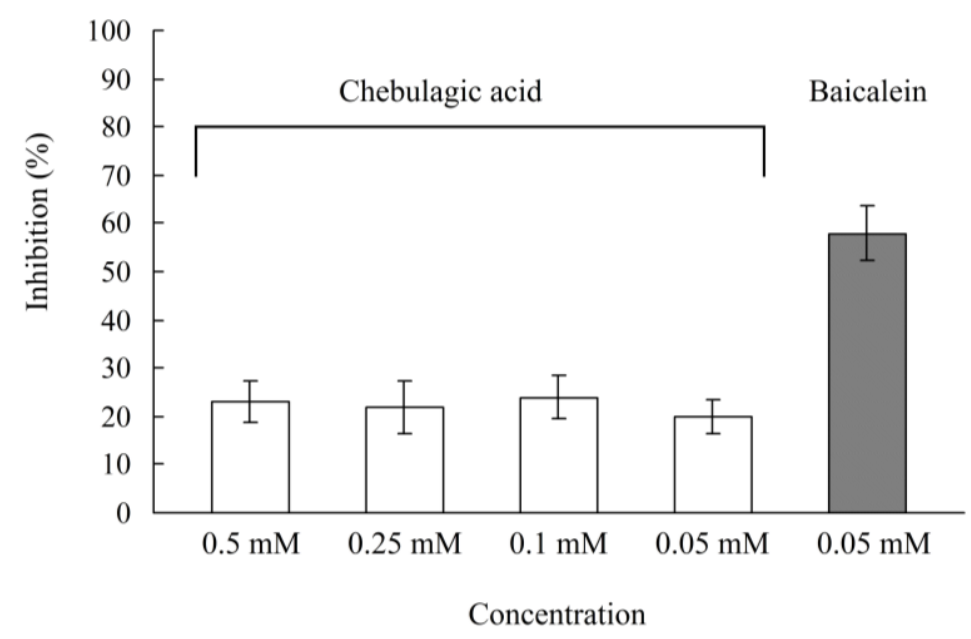

In our previous study, analysis of a Lineweaver-Burk plot of rat intestinal maltase kinetics suggested that chebulagic acid shows a non-competitive inhibition, in which chebulagic acid and the maltose substrate seem to bind simultaneously to the enzyme [17]. In order to determine whether chebulagic acid is a reversible maltase inhibitor, Caco-2 cells were pretreated by chebulagic acid (0.05-0.5 mM/well levels) for $20 \mathrm{~min}$ at $37{ }^{\circ} \mathrm{C}$ in $5 \% \mathrm{CO}_{2}$ atmosphere. Then it was examined whether the maltose-hydrolysis activity would be influenced or not in the Caco-2 monolayer. Compared with the control, the maltose-hydrolysis activities were in the ranges of 95-98\% at the dose of $0.05-0.5 \mathrm{mM}$ /well levels pretreatment with chebulagic acid (Figure 5). It is obvious that chebulagic acid is a reversible inhibitor of maltase.

Figure 5. Maltose-hydrolysis activity in the Caco-2 monolayer pretreated with chebulagic acid $(0.05-0.5 \mathrm{mM} /$ well $)$ for $20 \mathrm{~min}$ at $37^{\circ} \mathrm{C}$ in $5 \% \mathrm{CO}_{2}$ atmosphere.

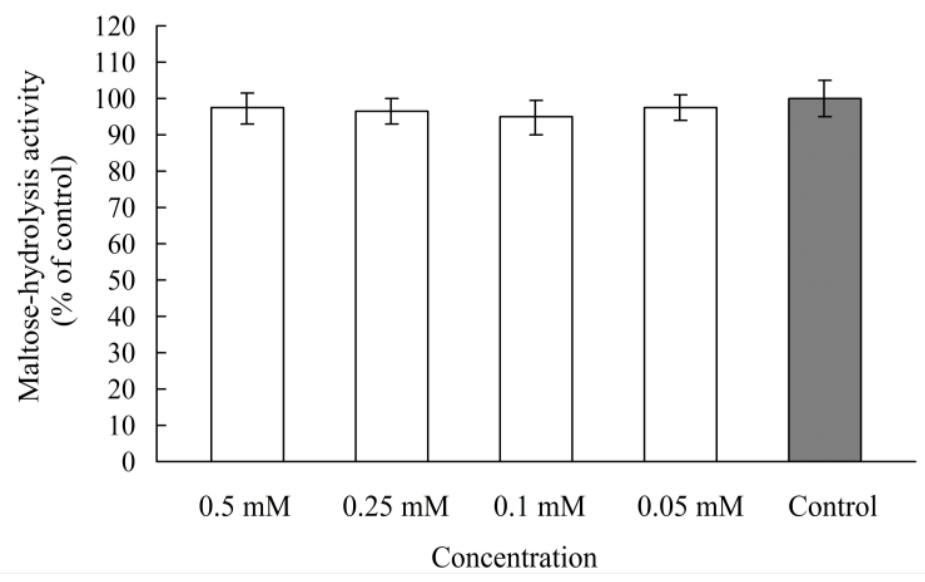

The liberated glucose from carbohydrate digestion is absorbed across the intestinal enterocytes via specific transporters, so glucose absorption is a potent target for better glycemia control after high-carbohydrate meals. Inhibition of glucose transporters would reduce the absorption into the small intestine and consequently suppress postprandial hyperglycemia [24]. A previous study 
reported that dietary polyphenols, such as (-)-epigallochatechingallate, (-)-epichatechingallate and (-)-epigallochatechin, are effective against glucose transporters and decrease glucose uptake by human intestinal Caco-2 cells [25]. In the present study, the effect of chebulagic acid on intestinal glucose uptake was investigated by using Caco-2 monolayers. As shown in Figure 6, compared with the control (glucose accumulation at the basal sides of the Caco- 2 monolayer as $100 \%$ uptake), the glucose uptake rates were in the ranges of $94-98 \%$ in the presence of chebulagic acid $(0.05-0.5 \mathrm{mM} / \mathrm{well})$. This result suggests that chebulagic acid does not have an obvious influence on intestinal glucose uptake and has no effect on glucose transporters.

Figure 6. Glucose uptake in the Caco-2 monolayer in the presence of chebulagic acid $(0.05-0.5 \mathrm{mM} / \mathrm{well})$.

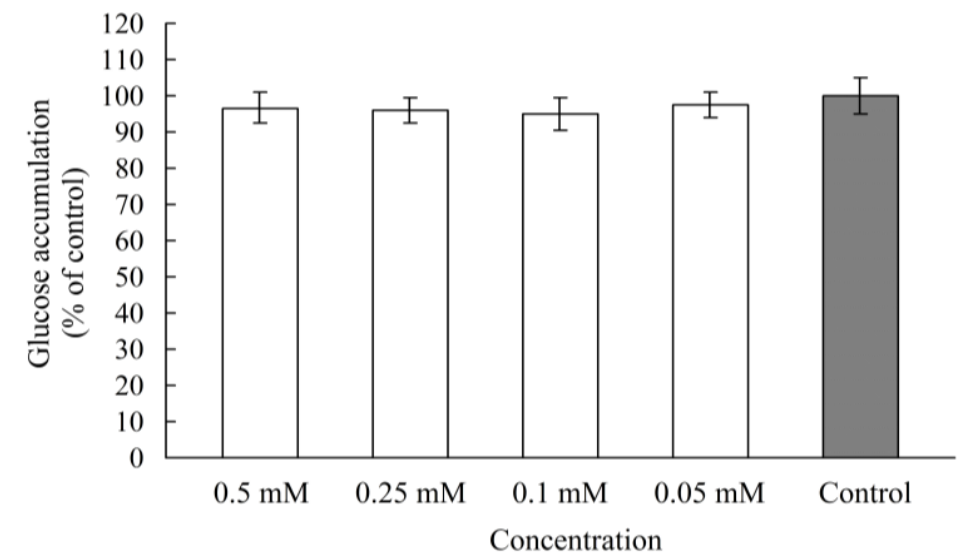

\subsection{Effect of Chebulagic Acid on Blood Glucose Level in Sugar-Loaded SD-Rats}

Our previous study showed that the oral administration of $T$. chebula fruit extract significantly reduced postprandial blood glucose levels in SD-rats after oral loads of maltose [26]. To clarify the active compound associated with the suppression of the rat blood glucose level, an anti-hyperglycemic effect of chebulagic acid in the extract was investigated.

Figure 7 shows the anti-hyperglycemic effects of chebulagic acid in maltose (A)-, sucrose (B)-, and glucose (C)-loaded SD-rats. In the maltose-loading test, the blood glucose level in the chebulagic acid group was significantly lower than that in the control group (Figure 7A). The blood glucose level $30 \mathrm{~min}$ after administration was $126.7 \pm 5.6 \mathrm{mg} / \mathrm{dL}$ in chebulagic acid (100 mg/kg, body weight) group, while in the control group the value was $165.2 \pm 8.4 \mathrm{mg} / \mathrm{dL}$. The raise in blood glucose level was reduced by $23.3 \% 30 \mathrm{~min}$ after gavage. The blood levels of chebulagic acid administrated SD-rats at 60 and $120 \mathrm{~min}$ were $120.2 \pm 7.2$ and $72.1 \pm 6.9 \mathrm{mg} / \mathrm{dL}$, respectively, while the values in the controls were $126.4 \pm 4.8$ and $77.0 \pm 9.1 \mathrm{mg} / \mathrm{dL}$, respectively. The area under the glycemic curve $\left(\mathrm{AUC}_{0-120 \mathrm{~min}}\right)$ for chebulagic acid administrated SD-rats $(207.8 \pm 7.9 \mathrm{mg} \cdot \mathrm{h} / \mathrm{dL}$ of blood) showed a significant reduction of $11.1 \%$ compared with that of the control group $(233.64 \pm 6.6 \mathrm{mg} \cdot \mathrm{h} / \mathrm{dL}$ of blood). The acute intake of acarbose reduced the incremental $\mathrm{AUC}_{0-120 \mathrm{~min}}(199.6 \pm 7.5 \mathrm{mg} \cdot \mathrm{h} / \mathrm{dL}$ of blood $)$ in the SD-rats after maltose administration by $14.6 \%$. Our animal study showed that the oral administration of chebulagic acid had a suppressive effect on hyperglycemia, which was similar to that seen with the administration of $3 \mathrm{mg} / \mathrm{kg}$ acarbose. 
On the other hand, in the sucrose-loading test, the blood glucose level in the chebulagic acid (100 mg/kg, body weight) group was not significantly different from that of the control group (Figure 7B). As in vitro Caco-2 sucrase inhibitory activity of chebulagic acid was weak (Figure 4), there seemed to be no blood glucose level reduction in the sucrose-loaded SD-rats by chebulagic acid. The result was similar to the previous report in which methanol extracts of dried flowers of Ranawara (Cassia auriculata) with a potent maltase inhibition did not significantly reduce the postprandial blood glucose levels in sucrose-loaded rats, because Ranawara showed a weak sucrase inhibitory activity [27].

A glucose-loading test was also performed at a dose of $100 \mathrm{mg} / \mathrm{kg}$. As shown in Figure 7C, the administration of chebulagic acid did not affect postprandial blood glucose levels in SD-rats after oral-loading glucose. According to the result obtained in glucose uptake by the Caco-2 monolayer (Figure 6), no blood glucose level reduction in glucose-loaded rats by chebulagic acid indicated that the significant hypoglycemic effect in maltose-loaded SD-rats resulted from intestinal maltase inhibition, not from intestinal glucose transporter inhibition.

Figure 7. Anti-hyperglycemic effects of chebulagic acid on SD-rats. Fasted rats were given $2 \mathrm{~g} / \mathrm{kg}$ of maltose (A), $2 \mathrm{~g} / \mathrm{kg}$ of sucrose (B) and $2 \mathrm{~g} / \mathrm{kg}$ of glucose (C), with or without $100 \mathrm{mg} / \mathrm{kg}$ of chebulagic acid ( $\mathbf{n})$ and vehicle (control: $\downarrow$ ). Acarbose (०) was used as a positive control for maltose-loaded SD-rats (A). Data are presented as the mean \pm SD $(n=5) . * p<0.05 v s$. control.

(A)

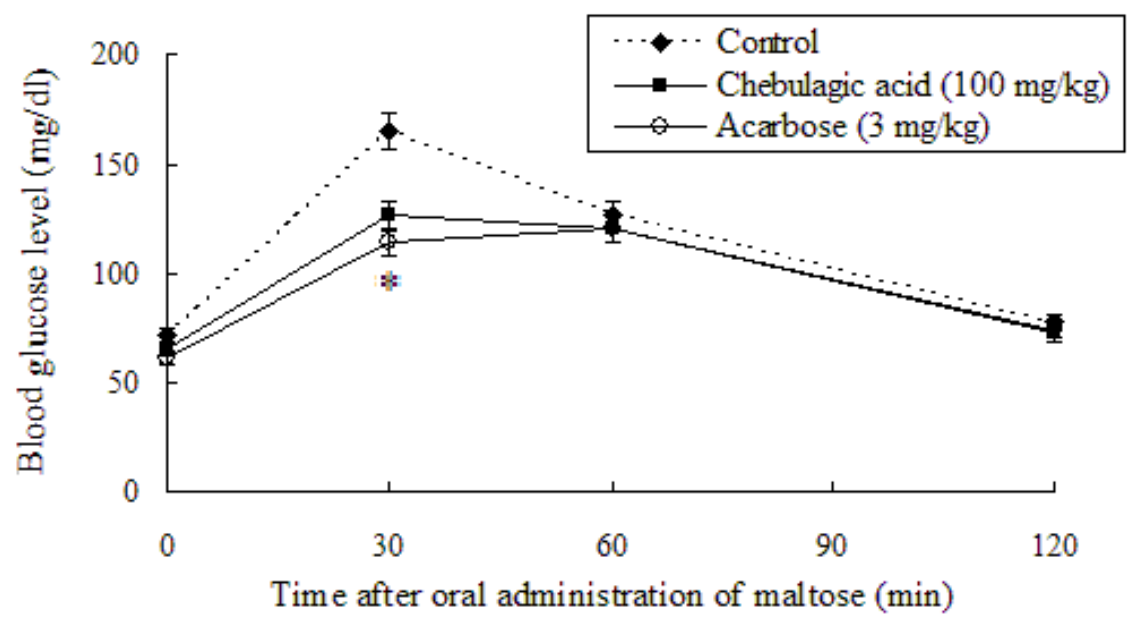

(B)

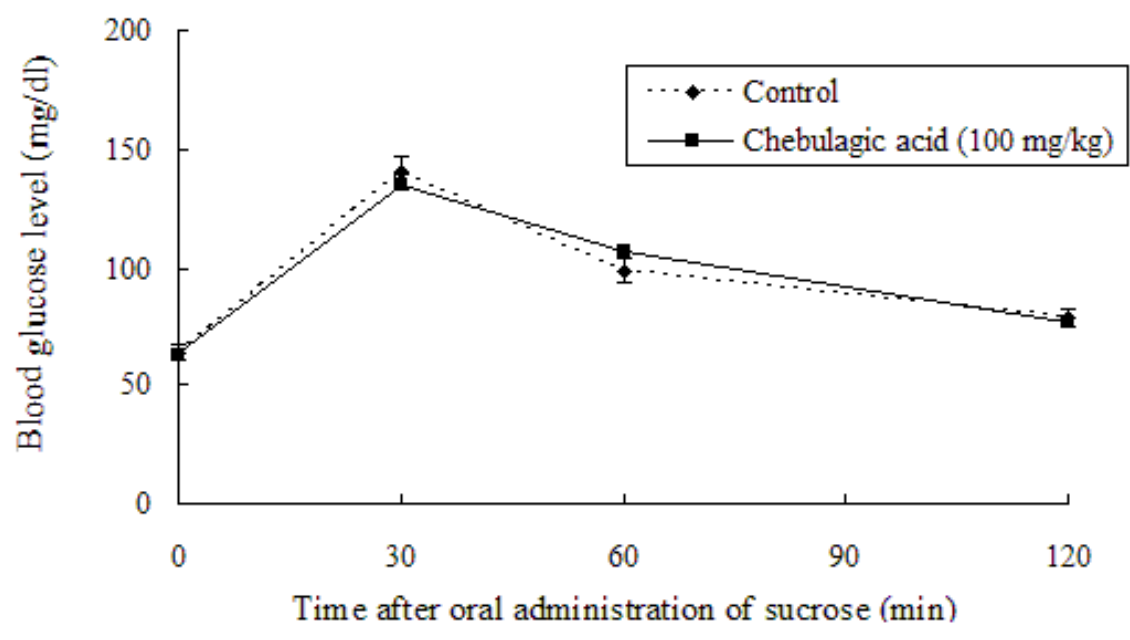


Figure 7. Cont.

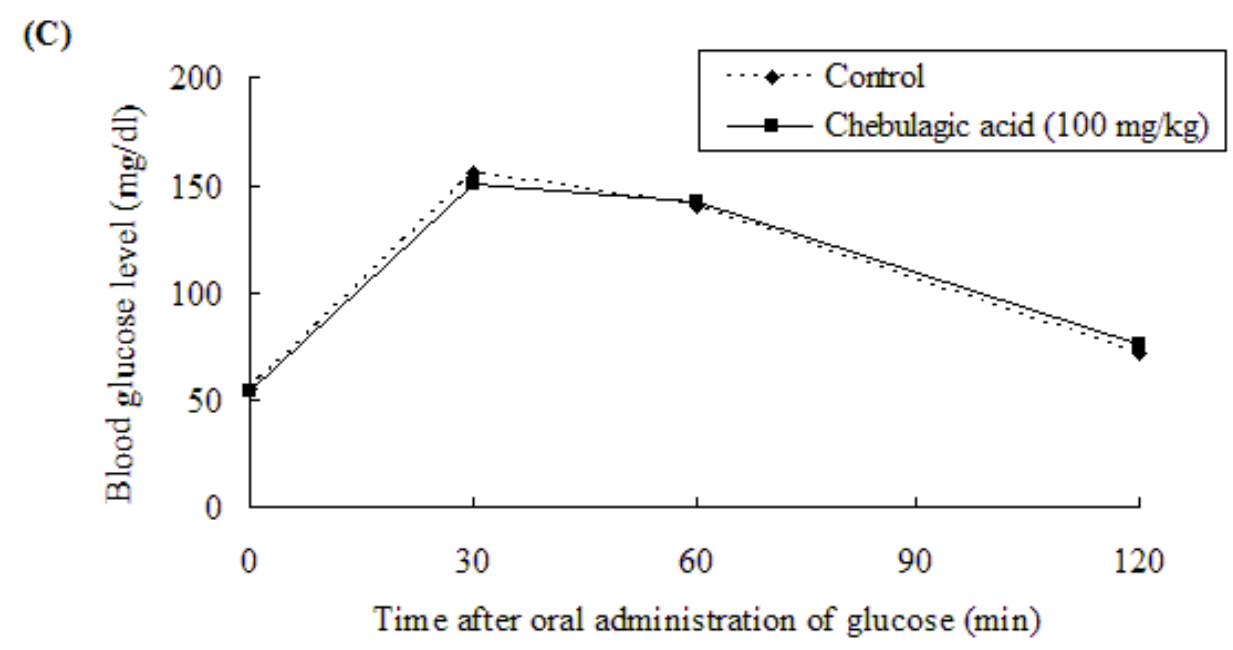

\section{Materials and Methods}

\subsection{Materials}

The dried fruits of $T$. chebula were purchased from a local herbal market in Chengdu, China, and properly identified at the Department of Pharmacology, Hua Xi Medicinal Center of Sichuan University. The voucher specimen (No. 2011070202) is deposited in the department of Public Health, Hua Xi Medical Center of Sichuan University. Maltose was purchased from Merck Chemical Supplies. All other chemicals were purchased from Sigma-Aldrich Company unless otherwise stated. All other chemicals used including the solvents were of an analytical grade.

\subsection{Preparation of Sample Extraction}

The dried fruits of $T$. chebula were powdered in a blender. For extraction, every gram of the powered fruits was suspended in $20 \mathrm{~mL}$ of an extraction solvent: One hundred percent ethanol, 80\% ethanol $($ ethanol $/$ water $=8: 2, \mathrm{v} / \mathrm{v})$ or $50 \%$ ethanol $($ ethanol $/$ water $=1: 1, \mathrm{v} / \mathrm{v})$. Extractions were carried out at room temperature for $36 \mathrm{~h}$ with continuous stirring and then treated with ultrasonication for $1 \mathrm{~h}$. After filtration, the solvent from this extract was evaporated and the residues were lyophilized. The lyophilized powders were stored at $-20^{\circ} \mathrm{C}$ before use.

\subsection{Determination of Chebulagic Acid in Each Extract}

An amount of $10 \mathrm{mg}$ of each of the above-mentioned extracts was dissolved in $1 \mathrm{~mL}$ of $70 \%$ methanol (a final concentration of $10 \mathrm{mg} / \mathrm{mL}$ ), which was then passed through a HP020 filter (Advantec). Then $20 \mu \mathrm{L}$ of this filtrate was injected directly for reverse-phase-HPLC analysis. The HPLC system (Agilent 1200 Series Purification System) consisted of an injector (G1328B), a column oven $\left(25{ }^{\circ} \mathrm{C}\right)$, a pump (G1311A), a diode array detector (G1315D), and a reverse-phase column (Inertsil PREP-ODS-3, $4.6 \times 250 \mathrm{~mm}, 5 \mu \mathrm{m}$, GL-Science). A linear gradient elution system, using solvent $\mathrm{A}$ and $\mathrm{B}(\mathrm{A}=$ methanol; $\mathrm{B}=0.1 \%$ formic acid in ultrapure water) according to the following profile: 0-10 min, 10-20\% A, 90-80\% B; 10-30 min, 20-30\% A, 80-70\% B; 30-50 min, 30-40\% A, 
70-60\% B; 50-70 min, 40-50\% A, 60-50\% B; 70-90 min, 50-100\% A, 50-0\% B). The flow rate was maintained constant at $1.2 \mathrm{~mL} / \mathrm{min}$, and the phenolic compounds were monitored at $280 \mathrm{~nm}$.

\subsection{Isolation of Chebulagic Acid}

Chebulagic acid was prepared with our previous reported method [17] except that the dried fruit powders of $T$. chebula were extracted with $80 \%$ ethanol instead of $70 \%$ methanol. The chemical structure of chebulagic acid was identified with ${ }^{1} \mathrm{H}-\mathrm{NMR}$ and MS and the data were compared with data in the literature [17].

\subsection{Intestinal $\alpha$-Glucosidase Inhibitory Activity}

The $\alpha$-glucosidase inhibitory activity was measured as described previously [17]. The crude enzyme solution prepared from rat intestinal acetone powder (Sigma-Aldrich) was used as the small intestinal $\alpha$-glucosidase. The reaction mixture consisted of a crude enzyme solution $(0.05 \mathrm{~mL})$, a substrate (maltose, $3.5 \mathrm{mM}, 0.35 \mathrm{~mL}$ ) in $0.1 \mathrm{M}$ potassium phosphate buffer ( $\mathrm{pH}$ 6.3), and the test sample in $50 \%$ DMSO $(0.1 \mathrm{~mL})$. After incubation for $15 \mathrm{~min}$ at $37{ }^{\circ} \mathrm{C}$, the reaction was stopped by adding $0.75 \mathrm{~mL}$ of $2 \mathrm{M}$ Tris- $\mathrm{HCl}$ buffer ( $\mathrm{pH}$ 7.0). The reaction mixture was passed through a short column of basic alumina, and the amount of liberated glucose was measured by the glucose oxidase method. The concentration of inhibitors required for inhibiting $50 \%$ of the $\alpha$-glucosidase activity under the assay conditions was defined as the $\mathrm{IC}_{50}$ value.

\subsection{Assay for the Caco-2 Cells Experiment}

The intestinal epithelial cell line, Caco-2, derived from a human colon adenocarcinoma was used as an intestinal membrane model. Caco-2 cells used in this study were obtained from the National Chengdu Center for Safety Evaluation of Drugs. Cell viability was estimated by the MTT (3-(4,5-dimethylthiazol-2-yl)-2,5-diphenyltetrazolium bromide) assay. Briefly, Caco-2 cells were seeded in a 96 well plate at $1 \times 10^{5}$ cells $/ \mathrm{mL}$. Twenty-four hours after plating, cells were treated with various concentrations of test sample and were incubated for an additional $2 \mathrm{~h}$ at $37{ }^{\circ} \mathrm{C}$. After the cell culture medium was removed, the cells were incubated with $20 \mu \mathrm{L}$ of MTT solutions $(5 \mathrm{mg} / \mathrm{mL})$ in $100 \mu \mathrm{L}$ of medium at $37{ }^{\circ} \mathrm{C}$ for $4 \mathrm{~h}$. The insoluble derivative formed by cellular dehydrogenase was solubilized with DMSO and absorbance was measured at $490 \mathrm{~nm}$ with a microplate reader.

Alpha-Glucosidase inhibitory activity in Caco-2 cell lines was performed with the previous method [28]. Briefly, Caco- 2 cells were fed on polyethylene terephthalate membranes (Falcon, pore size: $0.4 \mu \mathrm{m}$, pore density $1.6 \times 10^{6}$ pores $/ \mathrm{cm}^{2}$, diameter: $23.1 \mathrm{~mm}$ ) in a 6 well plate. After 16 days, the cell culture medium was removed and both the apical and basal chambers were washed 3 times with $2 \mathrm{~mL}$ of phosphate-buffered saline (PBS). The culture medium in the apical chamber was replaced with a reaction mixture containing chebulagic acid $(0.05 \mathrm{~mL})$ and a sugar solution $(28 \mathrm{mM}$ maltose or $28 \mathrm{mM}$ sucrose) in PBS $(0.95 \mathrm{~mL})$ as a substrate. In the basal chamber $1 \mathrm{~mL}$ of PBS was added instead of the culture medium. After the Caco-2 enzyme reaction at $37{ }^{\circ} \mathrm{C}$ in $5 \% \mathrm{CO}_{2}$ atmosphere for $2 \mathrm{~h}, 500 \mu \mathrm{L}$ of each bathing solution on the apical side was transferred to pass through a short column of basic alumina, and the content of liberated glucose was determined by the glucose oxidase method. 
The effect of chebulagic acid on $\alpha$-glucosidase inhibitory activity was observed in Caco- 2 cell lines. Briefly, Caco-2 cells $\left(1.6 \times 10^{6} / \mathrm{mL}\right)$ were fed onto a 12 well plate. After 16 days, the cell culture medium was removed and cells were treated with chebulagic acid in $1 \mathrm{~mL}$ of PBS. After $20 \mathrm{~min}$ incubation at $37{ }^{\circ} \mathrm{C}$ in $5 \% \mathrm{CO}_{2}$ atmosphere, the treatment solution in each well was removed and the cells were washed twice with PBS. Then, the maltose-hydrolysis activity in the Caco-2 cells was determined as described.

The effect of chebulagic acid on glucose uptake was tested with Caco- 2 cells lines in a 6 well plate. After 16 days, the cell culture medium was removed and both the apical and basal chambers were washed 3 times with $2 \mathrm{~mL}$ of phosphate-buffered saline (PBS). The culture medium in the apical chamber was replaced with a reaction mixture containing chebulagic acid $(0.05 \mathrm{~mL})$ and $28 \mathrm{mM}$ glucose in PBS $(0.95 \mathrm{~mL})$. In the basal chamber $1 \mathrm{~mL}$ of PBS was added instead of the culture medium. After incubation at $37{ }^{\circ} \mathrm{C}$ in $5 \% \mathrm{CO}_{2}$ atmosphere for $2 \mathrm{~h}$, the glucose levels on the basal side were determined as described.

\subsection{Animal Experimental}

Six-week-old male Sprague-Dawley (SD) rats were purchased from the Laboratory Animal Center, Sichuan Academy of Medical Sciences. SD-rats were housed in an air-conditioned environment and with a $12 \mathrm{~h} \mathrm{light/dark}$ cycle. The temperature was maintained at $22 \pm 1{ }^{\circ} \mathrm{C}$ and the relative humidity was $55 \pm 10 \%$. The animals were provided with standard laboratory animal feed and water ad libitum. The SD-rats were fasted for $12 \mathrm{~h}$ and divided into groups according to body weight (200-250 g, $n=5$ ). Each sample was dissolved in water and administrated by gavage. In the control group, $1 \mathrm{~mL}$ of $2 \mathrm{~g} / \mathrm{kg}$ of a sugar (maltose, sucrose or glucose) solution was administrated to each rat. In the sample-treated group, SD-rats were given chebulagic acid (100 mg/kg body weight) or a positive control, acarbose $(3 \mathrm{mg} / \mathrm{kg}$ body weight). Ten minutes after oral administration, the sugar-loading test was done. Blood samples were collected from the tips of the rats' tails at 30, 60, 90 and 120 min after each sugar loading. The blood glucose levels were measured immediately with a commercial glucometer and test-strips (GT-1640, Kyoto, Japan).

All experiments were carried out in accordance with the ethical guidelines of the Sichuan Province Experimental Animal Management Committee and were in complete compliance with the National Institutes of Health Guide for the Care and Use of Laboratory Animals.

\subsection{Statistical Analysis}

Data were reported as mean \pm standard deviation of mean. Statistical analyses were performed with Student's $t$-test using the SPSS program (version 12.0 for Windows, SPSS Inc., Chicago, IL, USA). Values were considered to differ significantly if the $p$ value was less than 0.05 .

\section{Conclusions}

Mammalian intestinal $\alpha$-glucosidase inhibitors can reduce postprandial plasma glucose levels and absorption of starch and disaccharides, and play potent roles in modulating postprandial hyperglycemia. In a previous study, we reported that the methanol extract from the fruits of $T$. chebula showed a 
potent maltase inhibitory activity and the active compounds, chebulanin, chebulagic acid and chebulinic acid, were identified from this plant. In the present study, we compared rat intestinal $\alpha$-glucosidase inhibitory activities of different ethanol-aqueous extractions from the dried fruits of T. chebula. The enzymatic assay showed that the $80 \%$ ethanol extract had a more potent effect on maltase activity than both $50 \%$ and $80 \%$ ethanol extracts. HPLC analysis revealed that the $80 \%$ ethanol extract contained a higher content of chebulagic acid than either the $50 \%$ or $100 \%$ ethanol extract. In a Caco-2 cell model, $\alpha$-glucosidase activity for maltose hydrolysis was down-regulated by chebulagic acid, which is a reversible inhibitor of maltase. On the other hand, chebulagic acid showed a weak inhibition of sucrose-hydrolysis activity and did not affect intestinal glucose uptake by Caco-2 cells. Furthermore, chebulagic acid significantly reduced postprandial blood glucose level in maltose-loaded SD-rats. So, it was suggested that chebulagic acid from T. chebula may be useful for suppressing postprandial hyperglycemia as a potent anti-diabetic agent, although clinical trials are needed.

\section{Acknowledgments}

This work was supported by the National Natural Science Foundation of China (grant number: 31071489) and the Research Fund of New Teachers for the Doctoral Program of Higher Education of China (No. 201001811220022).

\section{References}

1. Zhao, L.Y.; Lan, Q.J.; Huang, Z.C.; Ouyang, L.J.; Zeng, F.H. Antidiabetic effect of a newly identified component of Opuntia dillenii polysaccharides. Phytomedicine 2011, 18, 661-668.

2. Rendell, M. The role of sulphonylureas in the management of type 2 diabetes mellitus. Drugs 2004, 64, 1339-1358.

3. Lebovitz, H.E. alpha-Glucosidase inhibitors. Endocrinol. Metab. Clin. North Am. 1997, 26, 539-551.

4. Yao, Y.; Cheng, X.Z.; Wang, L.X.; Wang, S.H.; Ren, G.X. A determination of potential $\alpha$-glucosidase inhibitors from azuki beans (Vigna angularis). Int. J. Mol. Sci. 2011, 12, 6445-6451.

5. Kim, S.H.; Jo, S.H.; Kwon, Y.I.; Kwang, J.K. Effects of onion (Allium cepa L.) extract administration on intestinal $\alpha$-glucosidases activities and spikes in postprandial blood glucose levels in SD rats model. Int. J. Mol. Sci. 2011, 12, 3757-3769.

6. Gao, H.; Huang, Y.N.; Gao, B.; Xu, P.Y.; Inagaki, C.; Kawabata, J. $\alpha$-Glucosidase inhibitory effect by the flower buds of Tussilago farfara L. Food Chem. 2008, 106, 1195-1201.

7. Srivastava, P.; Raut, H.N.; Wagh, R.S.; Puntambekar, H.M.; Kulkarni, M.J. Purification and characterization of an antioxidant protein $(\sim 16 \mathrm{kDa})$ from Terminalia chebula fruit. Food Chem. 2012, 131, 141-148.

8. Chang, C.L.; Lin, C.S. Phytochemical composition, antioxidant activity, and neuroprotective effect of Terminalia chebula Retzius extracts. Evid. Based Complement. Altern. Med. 2012, 2012, doi:10.1155/2012/125247. 
9. Saleem, A.; Husheem, M.; Härkönen, P.; Pihlajia, K. Inhibition of cancer cell growth by crude extract and the phenolics of Terminalia chebula Retz. fruit. J. Ethnopharmacol. 2002, 81, 327-336.

10. Malekzadeh, F.; Ehsanifar, H.; Shahamat, M.; Levin, M.; Colwell, R.R. Antibacterial activity of black myrobalan (Terminalia chebula Retz) against Helicobacter pylori. Int. J. Antimicrob. Agents 2011, 18, 85-88.

11. Sabu, M.C.; Kuttan, R. Anti-diabetic activity of medicinal plants and its relationship with their antioxidant property. J. Ethnopharmacol. 2002, 81, 155-160.

12. Rao, N.K.; Nammi, S. Antidiabetic and renoprotective effects of the chloroform extract of Terminalia chebula Retz. seeds in streptozotocin-induced diabetic rats. BMC Complement. Altern. Med. 2006, 6, 17-22.

13. Murali, Y.K.; Anand, P.; Tandon, V.; Singh, R.; Chandra, R.; Murthy, P.S. Long-term effects of Terminalia chebula Retz. on hyperglycemia and associated hyperlipidemia, tissue glycogen content and in vitro release of insulin in streptozotocin induced diabetic rats. Exp. Clin. Endocrinol. Diabetes 2007, 115, 641-646.

14. Pfundstein, B.; Ei Desouky, S.K.; Hull, W.E.; Haubner, R.; Erben, G.; Owen, R.W. Polyphenolic compounds in the fruits of Egyptian medicinal plants (Terminalia bellerica, Terminalia chebula and Terminalia horrida): Characterization, quantitation and determination of antioxidant capacities. Phytochemistry 2010, 71, 1132-1148.

15. Kaur, S.; Grover, I.S.; Singh, M.; Kaur, S. Antimutagenicity of hydrolyzable tannins from Terminalia chebula in Salmonella typhimurium. Mutat. Res. 1998, 419, 169-179.

16. Kundu, A.P.; Mahato, S.B. Triterpenoids and their glycosides from Terminalia chebula. Phytochemistry 1993, 32, 999-1002.

17. Gao, H.; Huang, Y.N.; Xu, P.Y.; Kawabata, J. Inhibitory effect on $\alpha$-glucosidase by the fruits of Terminalia chebula Retz. Food Chem. 2007, 105, 628-634.

18. Gao, H.; Huang, Y.N.; Gao, B.; Kawabata, J. Chebulagic acid is a potent $\alpha$-glucosidase inhibitor. Biosci. Biotechnol. Biochem. 2008, 72, 601-603.

19. Tubtimdee, C.; Shotipruk, A. Extraction of phenolics from Terminalia chebula Retz with water-ethanol and water-propylene glycol and sugaring-out concentration of extracts. Sep. Purif. Technol. 2011, 77, 339-346.

20. Palanisamy, U.; Manaharan, T.; Teng, L.L.; Radhakrishnan, A.K.C.; Subramaniam, T.; Masilamani, T. Rambutan rind in the management of hyperglycemia. Food Res. Int. 2011, 44, 2278-2282.

21. Mahraoui, L.; Rodolosse, A.; Barbat, A.; Dussaulx, E.; Zweibaum, A.; Rousset, M. Presence and differential expression of SGLT1, GLUT1, GLUT2, GLUT3 and GLUT5 hexose-transporter mRNAs in Caco-2 cell clones in relation to cell growth and glucose consumption. Biochem. J. 1994, 298, 629-633.

22. Toda, M.; Kawabata, J.; Kasai, T. $\alpha$-Glucosidase inhibitors from Clove (Syzgium aromaticum). Biosci. Biotechnol. Biochem. 2000, 64, 294-298.

23. Hansawasdi, C.; Kawabata, J. $\alpha$-Glucosidase inhibitory effect of mulberry (Morus alba) leaves on Caco-2. Fitoterapia 2006, 77, 568-573. 
24. Hanhineva, K.; Törrönen, R.; Bondia-Pons, I.; Pekkinen, J.; Kolehmainen, M.; Mykkänen, K.; Poutanen, K. Impact of dietary polyphenols on carbohydrate metabolism. Int. J. Mol. Sci. 2010, $11,1365-1402$.

25. Johnston, K.; Sharp, P.; Clifford, M.; Morgan, L. Dietary polyphenols decrease glucose uptake by human intestinal Caco-2 cells. FEBS Lett. 2005, 579, 1653-1657.

26. Jing, Z.; Zeng, W.C.; Luo, J.W.; Ye, H.X.; Huang, Y.N.; Gao, H. In vitro and in vivo inhibitory effect of methanol extract from Terminalia chebula Retz. fruits on $\alpha$-glucosidase (In Chinsese). Food Sci. 2010, 31, 284-287.

27. Abesundara, K.J.M.; Matsui, T.; Matsumoto, K. $\alpha$-Glucosidae inhibitory activity of Sri Lanka plant extracts, one of which, Cassia auriculata, exerts a strong antihyperglycemic effect in rats comparable to the therapeutic drug acrabose. J. Agric. Food Chem. 2004, 52, 2541-2545.

28. Huang, Y.N.; Zhao, Y.L.; Gao, X.L.; Zhao, Z.F.; Jing, Z.; Zeng, W.C.; Yang, R.; Peng, R.; Tong, T.; Wang, L.F.; Cen, J.Q.; Gao, H. Intestinal $\alpha$-glucosidase inhibitory activity and toxicological evaluation of Nymphaea stellata flowers extract. J. Ethnopharmacol. 2010, 131, 306-312.

(C) 2012 by the authors; licensee MDPI, Basel, Switzerland. This article is an open access article distributed under the terms and conditions of the Creative Commons Attribution license (http://creativecommons.org/licenses/by/3.0/). 